\title{
TRIMETHOPRIM-SULFAMETHOXAZOLE AND SODIUM VALPROATE-INDUCED TOXIC EPIDERMAL NECROLYSIS: A CASE SERIES
}

\author{
BHAVANA SRIVASTAVA, RENU KHANCHANDANI, VIKRAM SINGH DHAPOLA, ZAFAR MASOOD ANSARI*
}

Department of Pharmacology, Government Medical College, Haldwani, Nainital, Uttarakhand, India. Email: drzmasood@gmail.com

Received: 15 May 2021, Revised and Accepted: 14 July 2021

\section{ABSTRACT}

Toxic epidermal necrolysis (TEN) is a rare and serious but life-threatening dermolytic cutaneous reaction characterized by diffuse and severe exfoliation and destruction of the epidermis of skin and mucosa due to immunological damage of the epidermis which can bring about sepsis and respiratory distress. Drugs are the most common inflicting agents in the generation of TEN. Among drugs, antiepileptics, antipsychotics, and sulfadrugs are common causes of TEN. Valproate is one of the most common drugs prescribed for epilepsy, was found as causative agent in TEN in very few cases. Among sulfonamides, sulfamethoxazole is commonly used antibiotic which can cause TEN. The evidence-based treatment guidelines are lacking, so the best approach is to recognize and evade potential risk factors and to deliver intensive supportive care immediately to reduce morbidity and mortality. The aim of this case series is to focus on valproate and trimethoprim-sulfamethoxazole (TMP-SMX)-induced TEN, which are commonly used drugs. Here, we present a case series of TEN inflicted by TMP-SMX and sodium valproate in a 23-year-old female and 10-year-old boy, respectively, with successful recovery.

Keywords: Toxic epidermal necrolysis, Sulfamethoxazole, Sodium valproate, Immunological damage.

(C) 2021 The Authors. Published by Innovare Academic Sciences Pvt Ltd. This is an open access article under the CC BY license (http://creativecommons.org/ licenses/by/4.0/) DOI: http://dx.doi.org/10.22159/ajpcr.2021v14i8.42417. Journal homepage: https://innovareacademics.in/journals/index.php/ajpcr

\section{INTRODUCTION}

Toxic epidermal necrolysis (TEN) is a rare dermatological condition presenting with characteristic erythematous maculopapular rashes progressing into diffuse exfoliation of skin and mucous membranes along with constitutional symptoms and internal organ involvement [1]. TEN usually represents a drug-induced, idiosyncratic reaction, but may result from a variety of infections including HIV, Mycoplasma pneumoniae, or may have an unknown etiology [2]. The incidence is about $0.4-1.2$ cases/million person-years, but the mortality can be as high as $30-40 \%$ [3].

A variety of drugs can cause TEN; most commonly implicated ones are nonsteroidal anti-inflammatory drugs, sulfonamides and anticonvulsants [2]. Trimethoprim-sulfamethoxazole (TMP-SMX) is a widely used antibiotic and is often recommended for the treatment of many bacterial infections including Pneumocystis carinii pneumonia and urinary tract infections. Epilepsy and seizure are not uncommon problems in general populations in India. Valproate is one of the most commonly prescribed drugs for various types of epilepsy and seizure disorders. However, very few cases of drug-induced TEN have been reported due to these two inflicting drugs. These two are commonly prescribed drugs, so it is very important that prescribing doctors should be aware of this severe and dangerous but potentially curable adverse effect. Early recognition, intervention, and treatment of this serious adverse effect in time are the gold standard and can modify the progression of the disease condition and save the precious life of a patient.

In this case series, we present first case of a 23-year-old female who developed TEN without any prodromal symptoms following oral dose of TMP-SMX. Our second case in the series is a 10-year-old boy diagnosed with seizure disorder and developed TEN following valproate therapy.

\section{Consent from patients}

Both the patients of this case series gave consent for manuscript preparation and submission.

\section{CASE PRESENTATIONS}

\section{Patient 1}

A 23-year-old woman came to the outpatient department (OPD) of skin with diffuse erythematous rashes over her body following oral dose of TMP-SMX given by local practitioner for her burning micturition with fever. These lesions started as painful erythematous macules, progressed into blisters followed by diffuse exfoliation of the skin involving bilateral lower extremities, forearms, back, and abdomen. The symptoms developed within a few hours. She was already on antiretroviral therapy since long time consists of tenofovir, lamivudine, and nevirapine. She denied any known drug allergy. She also denied taking any herbal medications or drug of alternative medicine.

At presentation in OPD, she was afebrile, tachycardic with a pulse rate of $104 / \mathrm{min}$ and a blood pressure of 108/74 mmHg. Physical examination was remarkable for multiple areas of redness, blisters, and extensive detachment of skin involving $40 \%$ body surface area. Oral cavities and face were spared. Nikolsky sign was positive in the patient.

Laboratory investigations were significant for a white cell count of $6100 / \mu \mathrm{L}$ with normal differential leukocytes count, platelet count of $225,000 / \mu \mathrm{L}$, hemoglobin of $11.4 \mathrm{~g} / \mathrm{dL}$, and ESR was $16 \mathrm{~mm} / \mathrm{hr}$. Serum bicarbonate was $24 \mathrm{mmol} / \mathrm{L}$. Serum electrolytes, creatinine, urea, liver function test, partial thromboplastin time, prothrombin time, and international normalized ratio (INR) were also within normal range. A punch skin biopsy was obtained. Urine, blood, and wound cultures sent. Chest X-ray was done and it was normal.

\section{Patient 2}

A 10-year-old boy presented with an extensive exfoliation of skin involving maximum part of the body 6 days after initiation of sodium valproate therapy for his seizure disorder. This patient also had denudation of lip and oral mucosa. These lesions started as painful erythematous macules, rapidly progressed into multiple blisters further followed by diffuse exfoliation of the skin involving forearms, abdomen, and bilateral lower extremities. This pediatric patient was diagnosed as a case of epilepsy of recent onset and had been started on therapy 
with sodium valproate 1 week ago. On admission, the patient's vital signs included temperature $99 \mathrm{~F}$, pulse rate $122 / \mathrm{min}$, blood pressure $112 / 86 \mathrm{mmHg}$, and respiratory rate $21 / \mathrm{min}$. Approximately $50 \%$ of total body surface area affected with positive Nikolsky sign.

The laboratory investigations on admission showed $\mathrm{Hb}$ $12.4 \mathrm{gm} / \mathrm{dl}$, hematocrit $32.4 \%$, leukocyte count $10,700 / \mu \mathrm{L}$, urea $17.5 \mathrm{mg} / \mathrm{dL}$, and creatinine $0.8 \mathrm{mg} / \mathrm{dL}$. Serum bicarbonate was $25 \mathrm{mmol} / \mathrm{L}$. Serum electrolytes were within normal limits. Serum glutamic-pyruvic transaminase and serum glutamic-oxaloacetic transaminase were $60 \mathrm{U} / \mathrm{L}$ and $165 \mathrm{U} / \mathrm{L}$, respectively. Urine routine microscopic examination, blood cultures, and chest X-ray were also performed and did not reveal anything abnormal.

\section{DIFFERENTIAL DIAGNOSIS}

Differential diagnosis in the case of diffuse exfoliation of skin with multiple blisters includes TEN/Stevens-Johnson syndrome (SJS), toxic shock syndrome (TSS), staphylococcal scalded skin syndrome (SSSS), and bullous disorders of skin. Usually, patients of TSS and SSSS are febrile with an increased leukocyte count which was not found in these two patients. Later on, diagnosis of TEN was confirmed in our patients based on skin biopsy findings which were typical for TEN. Bullous disorders such as pemphigus and pemphigoid could be ruled out based on negative immunofluorescence on skin biopsy.

\section{TREATMENT}

These patients were admitted in the burn unit of a tertiary care teaching hospital. TMP-SMX and sodium valproate were stopped immediately in a 23-year-old female and 10-year-old boy, respectively. These patients were managed and treated with prophylactic antibiotics, intravenous fluid therapy, proper aseptic wound care, nutritional support, and analgesia. The aseptic wound care measures included initial debridement of necrotic tissue and desloughing followed by daily paraffin soaked gauze.

Electrolytes were replaced as and when needed. Both the patients were monitored continuously for their hemodynamic stability and healing of wounds. On the review of skin biopsy, we found full-thickness epidermal necrosis with blister formation in subepidermis and the presence of lymphocytes in perivesicular space, as well as dermal inflammation. Immunofluorescence test was negative in both the patients for basilar deposits. These findings were consistent with TEN.

SCORTEN disease severity scoring system (Table 1) can be used to evaluate prognosis in patients with SJS/TEN [4]. The validated SCORTEN disease severity scoring system can be used as a risk stratification tool to assess the severity and prognosis of TEN.

On the basis of SCORTEN variables, the SCORTEN value was found 1 and 2 in the first and second patient, respectively, in this case series.

Table 1: SCORTEN; TEN-specific severity of illness scoring system with respective mortality rates

\begin{tabular}{ll}
\hline SCORTEN variables & \\
Extent of epidermal detachment & $>10 \%$ \\
Age & $\geq 40$ years \\
Heart rate & $\geq 120 / \mathrm{min}$ \\
Bicarbonate & $<20 \mathrm{mmol} / \mathrm{L}$ \\
Serum urea nitrogen & $>28 \mathrm{mg} / \mathrm{dl}$ \\
Glucose & $>252 \mathrm{mg} / \mathrm{dl}$ \\
History of malignancy & \\
SCORTEN value predicted mortality rate $(\%)$ & \\
$0-1$ & 3.2 \\
2 & 12.1 \\
3 & 35.3 \\
4 & 58.3 \\
5 or more & $>90$ \\
\hline
\end{tabular}

On the basis of history of intake of inflicting drugs, thorough clinical examinations and pathological findings, these patients were diagnosed as a case of TEN which is due to sulfamethoxazole and sodium valproate intake in the first and second case, respectively.

\section{DISCUSSION}

TEN is a rare and severe but potentially fatal skin condition which is characterized by epidermal detachment, erythematous macules, bullous, and vesicular lesions involving predominantly skin and the mucous membranes [5].TEN is the part of a spectrum of dermatological conditions consist of three variants as per the involved body surface area (BSA); SJS $<10 \%$ BSA, SJS-TEN overlap condition 10-30\% BSA, and TEN $>30 \%$ BSA [6].

Maximum cases of TEN are due to intake of inflicting drugs and account for up to $80-95 \%$ of cases [2]. Nearly 200 different commonly used and prescribed drugs have been associated with TEN including antibiotics, anticonvulsants, allopurinol, nonsteroidal anti-inflammatory drugs, and steroids. Sulfa drugs are known to have the highest causative association with TEN among these medications and account for nearly $30 \%$ of the drug-induced cases [7]. The molecular mechanism by which sulfa drugs and other group of drugs causing TEN is unknown. It is believed to generate from an immunological reaction and process involving cytotoxic T cells, natural killer cells, and the generation of a cytotoxic protein called granulysin which is aimed at the destruction of the keratinocyte skin resulting in diffuse separation of epidermis from dermis [8].

The initial skin lesions in TEN consist of diffuse painful erythema followed by maculopapular lesions progressing to full-thickness sloughing of the epidermis and necrosis with diffuse exfoliation of skin. Mucosal involvement such as eyes, mouth, and vagina can occur. The characteristic finding in TEN is Nikolsky sign [2]. Denuded skin and damaged mucosa predispose to infection and dehydration due to loss of protective barrier leading to sepsis, respiratory distress, and shock [5]. The diagnosis of TEN is made predominantly clinically based on history of medicine exposure or intake, prodrome of flu-like symptoms, and illness and sloughing of necrotic skin lesions involving more than $30 \%$ BSA. It is further supported by histological findings, which includes fullthickness epidermal necrosis, subepithelial bullae, and separation of epidermis at dermal-epidermal junction with moderate infiltration of the upper dermis by mononuclear cells [9].

In the first case, there were signs and symptoms of TEN after taking TMPSMX. The lady was completely recovered after few weeks hospitalization by withdrawal of this offending drug, optimal care, and supportive treatment. In our second case, the offending drug was sodium valproate. This patient was also managed accordingly and recovered completely. The TEN typically heals slowly, taking 3-6 weeks. The recovery in TEN depends on the involved body surface area, depth of skin involvement, comorbid conditions, and any other associated complications that may occur during the course of illness. TEN and other burn-like syndromes are similar in many aspects to second-degree burns and the wound management is likewise similar to that of partial-thickness burns. The first line measures for TEN essentially include prompt identification and withdrawal of the offending $\operatorname{drug}(\mathrm{s})$, supportive therapy in an ICU setting, fluid/electrolytic and nutritional support, wound care as burns, and prevention and treatment of infection. The second line measures include a number of pharmacological therapies such as high-dose intravenous immunoglobulin (IVIG) therapy, intravenous steroids, and immunomodulating agents such as cyclophosphamide, ciclosporin, and plasmapheresis. However, with none of these therapies, there has been a consistent or conclusive evidence of benefit toward the patients' survival [10].

\section{CONCLUSION}

Although there was a common occurrence of adverse drug reaction in the form of TEN due to TMP-SMX as well as sodium valproate 
administration in two separate cases, we reported it to emphasize the important fact that extreme caution should be exercised while prescribing and administration of antimicrobial and anticonvulsant drugs, especially TMP-SMX and valproate to prevent this type of reaction. There was an involvement of the maximum parts of body skin and denudation of oral mucosa. The low SCORTEN score in our patient and their early presentation for optimum and supportive treatment contributed to the successful outcome in these cases. The doctors prescribing antibiotics specially sulfa drugs and antiepileptic agents to new users should be mindful of this potentially life threatening untoward side effect. Further studies are warranted to elucidate the relationship and the mechanism by which ofloxacin and chloroquine can cause Stevens-Johnson syndrome.

\section{ACKNOWLEDGMENT}

We are highly thankful to Pharmacovigilance Programme of India for their support, guidance, and contribution toward safety of the patients.

We are also highly obliged to these patients who gave consent for submission of this manuscript.

\section{CONFLICTS OF INTEREST}

Nil to all authors.

\section{AUTHORS' CONTRIBUTIONS}

Zafar Masood Ansari and Bhavana Srivastava contributed in writing the manuscript whereas Vikram Singh Dhapola and Renu Khanchandani provided the particulars and details of the patients.

\section{AUTHORS' FUNDING}

Authors have no funding.

\section{REFERENCES}

1. Gerull R, Nelle M, Schaible T. Toxic epidermal necrolysis and StevensJohnson syndrome: A review. Crit Care Med 2011;39:1521-32.

2. See S, Mumford JM. Trimethoprim/sulfamethoxazole-induced toxic epidermal necrolysis. Ann Pharmacother 2001;35:694-7.

3. Lissia M, Mulas P, Bulla A, Rubino C. Toxic epidermal necrolysis (Lyell's disease). Burns 2010;36:152-63.

4. Bastuji-Garin S, Fouchard N, Bertocchi M, Roujeau JC, Revuz J, Wolkenstein P. SCORTEN: A severity-of-illness score for toxic epidermal necrolysis. J Invest Dermatol 2000;115:149-53

5. Langlois MR, Derk F, Belczyk R, Zgonis T. Trimethoprimsulfamethoxazole-induced Stevens-Johnson syndrome: A case report. J Am Podiatr Med Assoc 2010;100:299-303.

6. Bastuji-Garin S, Rzany B, Stern RS, Shear NH, Naldi L, Roujeau JC. Clinical classification of cases of toxic epidermal necrolysis, StevensJohnson syndrome, and erythema multiforme. Arch Dermatol 1993;129:92-6.

7. Wolkenstein PE, Roujeau JC, Revuz J. Drug-induced toxic epidermal necrolysis. Clin Dermatol 1998;16:399-408.

8. Abe R, Yoshioka N, Murata J, Fujita Y, Shimizu H. Granulysin as a marker for early diagnosis of the Stevens-Johnson syndrome. Ann Intern Med 2009;151:514-5.

9. Rzany B, Hering O, Mockenhaupt M, Schröder W, Goerttler E, Ring J, et al. Histopathological and epidemiological characteristics of patients with erythema exudativum multiforme major, Stevens-Johnson syndrome and toxic epidermal necrolysis. Br J Dermatol 1996;135:6-11.

10. Hashim N, Bandara D, Tan E, Ilchyshyn A. Early cyclosporine treatment of incipient toxic epidermal necrolysis induced by concomitant use of lamotrigine and sodium valproate. Acta Derm Venereol 2004;84:90-1. 